\title{
Review
}

\section{Recent expeditious growth of type 1 diabetes in the Gulf Arab Countries}

\author{
Mohamed Jahromii, ${ }^{1,}$, Mona Al Sheikh ${ }^{2}$ and Jaakko Toumilehto ${ }^{3,4,5}$
}

Running title: Type 1 diabetes in the Gulf

1, " Correspondence : 1. Prime Autoimmunity Center, Manama, Bahrain; mjahromi@yahoo.com; +9773 33337437

2. Neurophysiology, College of Medicine, IAU, Dhahran, Saudi Arabia; monasheikh@iau.edu.sa

3. Public Health Promotion Unit, Finnish Institute for Health and Welfare, P.O. Box 30, 00271 Helsinki, Finlandjaakko.tumilehto@Th1.fi

4. Department of Public Health, University of Helsinki, 00014 Helsinki, Finland

5. Saudi Diabetes Research Group, King Abdulaziz University, 21589 Jeddah, Saudi Arabia

\begin{abstract}
The incidence of Type 1 Diabetes (T1D) in the Arab world, particularly, oil and gas rich Gulf Cooperative Council (GCC) countries has more than doubled in the last twenty years. Therefore, there is a dire need for careful systematic familial cohort studies, especially in high-risk populations. Several immunogenetic factors affect the pathogenesis of the disease. Genes in the human leukocyte antigen (HLA) account for the major genetic susceptibility to the disease. The triggering agents initiate disease onset by destruction of pancreatic $\beta$-cells. The autoantibodies against glutamic acid decarboxylase (GADA), insulinoma antigen-2 (IA-2A), insulin (IAA), and zinc transporter-8 (ZnT-8A) comprise the most reliable biomarkers for T1D in both children and adults. Although three of the GCC countries, namely Kuwait, Saudi Arabia and Qatar are among the top 10 countries with high incidence rate of T1D, no proper diagnostic and prediction tools were applied in the region. Understanding the disease sequelae in a homogenous gene pool with high consanguinity in the GCC could help solve the challenges in understanding pathogenesis, as well as hasten the prevention of T1D. Arab states must incorporate T1D predictive and intervention policies on a war-footing basis to minimize the burden of this serious disease.
\end{abstract}

Keywords: Type 1 diabetes, human leukocyte antigen, Kuwait Type 1 Diabetes Study, Islet autoantibodies, Insulin, prediction

\section{Introduction}

Type 1 diabetes [(T1D), MIM \#222100] is a common endocrine metabolic disease affecting children and adults across the world. It is a chronic immune-mediated disease that is characterized by insulin deficiency due to pancreatic $\beta$-cell destruction leading to hyperglycemia and is often associated with serious acute and chronic complications(1-3). As per the natural history of T1D, the initial non-symptomatic phase of the disease is triggered upon genetically susceptible individuals. During this phase of the disease $\beta$-cell autoimmunity and breakdown of $\beta$-cell-mass occurs as a result of biochemical reactions which lead to production of autoantibodies such as IA2, IAA, GADA, ZnT8 which are considered as current biomarkers for $\beta$-cell autoimmunity (Figure 1). Symptomatic disease usually appears in childhood or adolescence, although the symptoms can also develop much later in life(4). A recent study stated that the life expectancy of people with T1D is still approximately 12 years less than in the general population with reduced quality of life(5). As the causes and risk factors associated with T1D remain not fully understood, strategies for the cure and prevention developed until today have been unsuccessful. Patients with T1D are depending on a life-long insulin injection treatment (6). Recently, however, novel interventions approach to delay the symptomatic phase by repair of insulin gene, and finding neoepitopes of HLA responsible for $\beta$-cell immunity and 
autoantibody therapy are being tested but so far have been successful only in animal only. These efforts might prove to be useful in future.

The Gulf Cooperation Council, or GCC, is an intergovernmental organization made up of six member nations. This organization is also known as the Cooperation Council for the Arab States of the Gulf. This union is made up of almost all Arab states in the Gulf, except for Iraq. The nations that make up the GCC are Bahrain, Kuwait, Oman, Qatar, Saudi Arabia, and United Arab Emirates (UAE). Obesity in children, sedentary mode of life, lack of vitamin $\mathrm{D}$, have been proposed to be associated with the recent outbreak of T1D in the GCC (7)(8). Although intensive glycemic control can reduce the incidence of microvascular and macrovascular complications, most people with T1D still develop the latter complications(9). The nations that are members of the GCC make up to 2.6 million square Kilometers. The GCC territory house approximately four fifth of Arabian Peninsula, supports a population of over 54 million people, of whom $26 \%$ are aged 14 years or under (10). The total GDP of GCC nations is USD3.464 trillion. The GCC have undergone rapid economic growth and urbanization, associated with reduced infant mortality and increasing life expectancy. Studies in the recent decade indicate a significant increase in occurrence of T1D in Kuwait, Saudi Arabia and Qatar among the children and adults(11) (10)(12). Compared with Europe and the USA the knowledge about characteristics of T1D in Arabs is incomplete (13). In this review we discuss various aspects of T1D in the GCC in comparison with global approaches based on the currently available published literature.

T1D continues to surge despite several therapeutic advances and its incidence continues to be highly variable among countries. In 2020, the incidence rate varied by over 500-fold, with approximately 60/100,000 in Finland $(7,13)$ and 0.08/100,000 in Papua New Guinea $(13,14)$, Figure 2. It has been considered as a cold-area disease, in northern Europe and America. However, this does not hold true since for instance Sardinia in the Mediterranean has a very high incidence. Also, the incidence of T1D has shown a steep exponential growth in several Arab countries, especially oil-rich countries; the rate of T1D in Kuwait, Saudi Arabia and Qatar has experienced an over $100 \%$ increase in the last 20 years $(10-12,15)$. There are no published data about T1D incidence in Bahrain, Oman and UAE. Notably, all of GCC countries are among top 10 countries with high type 2 diabetes(11). If we presume that T1D comprises 5-10\% of total diabetes, eventually, T1D rates in Bahrain, Oman and UAE will not fall significantly from Kuwait, Saudi Arabia and Qatar. GCC countries comprise less than $1 \%$ of the world population, however, they carry a big proportion of the burden of this severe disease, Figure 2. In contrast, recent findings from Finland, Australia and Ireland suggest that the increasing trends have been declining or plateauing especially in children aged 4 years or under but not in older children $(16,17)$. The rates are higher in boys than girls in most high-incidence countries $(18,19)$. Europe has a good history of T1D registry systems in most countries developed since the late 1980s(20,21). Much of data about understanding of T1D has been derived from European and other western countries(20). In the Arab world, especially in the GCC region a good infrastructure for well-planned T1D research exist, but it is not organized properly. There are excellent scattered studies with lack of follow up or systematic efforts to maintain it. (22-27) .

Recently, it has been reported that T1D incidence is growing fast in the Middle East(8), and we have noted that the growth of T1D in the GCC has reached to an exponential growth compared with the rest of the world in less than 20 years $(11,12,15,28)$. Figure 2 illustrates the magnitude of the rate of T1D in Arab countries where data is available. This alarming increase could be multifactorial. High GDP has caused drastic change of lifestyle leading to obesity which is probably a risk factor for T1D. Other factors include lack of proper diagnostic regimens for T1D, diagnostic tool to distinguish Adult-Onset T1D (LADA) from T2D, systematic research, proper documentation and registry. In addition, consanguinity and endogamous marriages reported in the GCC countries are high, $23-64 \%(13,29)$ 
The genetic pool of the Arab populations in the GCC is relatively homogenous with a few subgroups $(13,29)$. Localizing genes and novel mutations in complex diseases have proven to be successful in such populations(13). Hence, there is an urgent demand on data from these unique GCC populations in order to fill gaps in international scientific understanding of the pathogenesis of T1D.

\section{Genetic studies in diabetes}

(13) Genes in the HLA region are explaining more than $50 \%$ of the genetic susceptibility in T1D. The HLA system (the major histocompatibility complex [MHC] in human) located on chromosome 6 is an important part of the immune system. It encodes cell surface molecules specialized to present antigenic peptides to the T-cell receptor (TCR) on T cells. HLA molecules that present antigen are divided into 3 main classes:

- $\quad$ Class I HLA: is encoded by genes at HLA-A, HLA-B, and HLA-C loci. T cells that express CD8 molecules react with class I HLA molecules. These lymphocytes often have a cytotoxic function, requiring them to be capable of recognizing any infected cell.

- $\quad$ Class II HLA: are usually present on Langerhans $\beta$-cells, thymic epithelium, and activated T cells; most nucleated cells can be induced to express class II MHC molecules by interferon (IFN)-gamma. Class II MHC molecules consist of 2 polypeptide $\alpha$ and $\beta$. Both polypeptide chains are encoded by genes in the HLA-DP, -DQ, or -DR region of chromosome 6 . T cells reactive to class II molecules express CD4 and are often helper cells.

- $\quad$ Class III HLA: region of the genome encodes several molecules important in inflammation; they include complement components $\mathrm{C} 2, \mathrm{C} 4$, and factor $\mathrm{B}$; tumor necrosis factor (TNF)-alpha; lymphotoxin; and three heat shock proteins.

Individual serologically defined antigens encoded by the class I and II gene loci in the HLA system are given standard designations (eg, HLA-A1, -B5, -C1, -DR1). Alleles defined by DNA sequencing are named to identify the gene, followed by an asterisk, numbers representing the allele group (often corresponding to the serologic antigen encoded by that allele), a colon, and numbers representing the specific allele (eg, $A^{*} 02: 01$, DRB1*01:03, DQA1*01:02). Sometimes additional numbers are added after a colon to identify allelic variants that encode identical proteins, and after another colon, other numbers are added to denote polymorphisms in introns or in $5^{\prime}$ or $3^{\prime}$ untranslated regions (eg, $\left.A^{*} 02: 101: 01: 02, D R B 1 * 03: 01: 01: 02\right)$. In Finland with the highest incidence of T1D the absolute risk for T1D for DR4,DQ8 positive haplotypes A2,C4,B35,DR4; A3,C3,B62,DR4; A24,C7,B39,DR4; A2,C3,B62,DR4, and A2,C1,B56,DR4 was 35, 130, 166, 196, and 218/100,000, respectively. The absolute risks for DR3,DQ2 positive haplotypes $\mathrm{A} 1, \mathrm{C} 7, \mathrm{~B} 8, \mathrm{DR} 3$, and A2,C7,B8,DR3 were 68 and 103/100,000, respectively. These results provide further evidence about polymorphism at the DQ locus but also other genes of the haplotypes contribute to susceptibility to $\mathrm{T} 1 \mathrm{D}(2,30,31)$

HLA class I and II molecules are the most immunogenic antigens that are well studied in T1D in Caucasians as well as Asians. The significance of associations in the HLA region with T1D diabetes were published in the 1970s $(32,33)$. HLA has been extensively studied for the last 50 years.

There is a dearth of studies on HLA among Arab populations $(13,34)$. Majority of studies on HLA in T1D in Arab populations have been carried out either on selected people and not well-specified populations and have concentrated on a few alleles rather than haplotypes. For example, there is no well-defined Arab HLA haplotype profile yet $(13,27)$. This is a woeful news since HLA haplotypes are strongly associated with T1D, and undoubtedly any future intervention, modification of stages of the disease or cure will be based on HLA.

Genes from the HLA region play multiple roles during immune response and they are the first checkpoints in its activation. Genetic polymorphisms encoding different amino acid residues in the peptide-binding pockets of HLA molecules are the main 
connection between HLA molecules and T1D. Moreover, the binding repertoire and affinity of peptides can be presented on T-cells $(2,35)$. The strongest association with T1D is located within the HLA class II region genes that encode highly polymorphic $\beta$-chains (HLADRB1, -DQA1 and -DQB1)(2). There are two main high-risk haplotypes "DR4-DQ8" (DR4DQA1*03:01-DQB1*03:02) and "DR3-DQ2" (DRB1*03:01-DQA1*05:01-DQB1*02:01). Around $90 \%$ of T1D patients carry DR4-DQ8 or DR3-DQ2 and roughly $30 \%$ patients carry the combination of both of those haplotypes (DR4-DQ8/DR3-DQ2). This group confers the highest risk of T1D development $(\mathrm{OR}=16)(2,36)$.

HLA class I alleles are also associated with T1D(37). In Finland with the highest incidence of T1D the absolute risk for T1D for DR4,DQ8 positive haplotypes varied significantly based on the class I gene loci. For instance, the incidence for A2,C4,B35,DR4; A3,C3,B62,DR4; A24,C7,B39,DR4; A2,C3,B62,DR4, and A2,C1,B56,DR4 was 35, 130, 166, 196 , and 218/100,000(37), when the overall incidence in the country was approximately 40/100,000. The absolute risks for DR3, DQ2 positive haplotypes A1, C7, B8, DR3, and A2,C7,B8,DR3 were 68 and 103/100,000, respectively. These results provide further evidence that not only the polymorphism at the DQ locus but also other genes of the haplotypes contribute to susceptibility to T1D; the entire HLA haplotype is the most important factor for the genetic predisposition(2,36). The presence of the HLA-B*39 allele has been shown to be connected with T1D diagnosis at young age(38). In addition, HLA-A*02 also increases the likelihood of T1D development and is one of the most frequent class I alleles, with frequency of $>60 \%$ in T1D patients (39). Table presents well known HLA haplotypes and diabetic risk values in different populations.

Recently we have established a well-planned Arab population familial-based study in Kuwait, Kuwaiti Autoimmune Diabetes Study (KADS)(13,40). We have carefully collected 56 Arab families with at least a single affected member. In order to confirm previously reported HLA data in Arab population we have recently accomplished our 11 loci HLA typing using the state of art technology of Next Genome Sequencing (NGS). We have come to extraordinary findings which have been published/or are in process $(25,41-43)$. We have come across unique Arab specific haplotypes, novel association of DRBs and DQBs in T1D. Having a homogenous rich gene pool, due to consanguinity and intermarriages among close relatives $(13,29)$, the above results confer the uniqueness of Arab, especially familial based studies, in revealing certain unknown facts about the etiology of the disease.

\section{Genetic susceptibility: HLA in Arab population}

Genetic susceptibility to T1D diabetes is determined by polymorphisms/ mutations in human genes $(4,36,44,45)$. More than 50 genes are known to influence the progression of T1D(6). Different HLA genotype patterns result in diverse rates of T1D among populations(46) and have the greatest impact compared to any other susceptibility genes. The associations of T1D with HLA class II have been shown to vary among different populations and ethnicities (Table 1) (47-50)For example, the high-risk HLA haplotypes in Caucasian populations, DRB1*03:01-DQB1*02:01 and DRB*04:01-DQB1*03:02, were found to be low in Japan and Southeast Asia with a low T1D incidence; instead, the susceptibility HLA haplotypes in Japanese and Korean populations were DRB1* 04:05-DQB1* 04:01 and DRB1* 09:01-DQB1*03:03(48). In Arab populations (i.e., Bahrainis, Lebanese, and Tunisians) who are primarily Caucasian, DRB1*03:01-DQB1*02:01 was reported (49), Table 1. In African Americans, the assessment of HLA risk differs significantly from that seen in other populations; both the DRB1*07:01 and DRB1*03:03 were high-risk haplotypes when DQA1*03:01-DQB1*02:01 was included(50). Interestingly, in African Americans, the DRB1*07:01-DQA1*02:01-DQB1*02:01g haplotype was not increased in European-derived T1D cases but was increased in African-derived cases(50). These studies conducted in homogeneous ethnic groups and comparing HLA DR-DQ-DP haplotypes offers evidence to link the risk of developing $\mathrm{T} 1 \mathrm{D}(51)$. They also provide evidence that the distribution of DP alleles varies depending on the ethnic group studied(52). Table 1 summarizes the 
classification of HLA-DR in different populations and their diabetes risk level $(2,38,44,46,47,52-62)$.

HLA genes are not transmitted randomly from parent to offspring, with solid linkage disequilibrium between A, C, B, DR and DQ alleles, i.e., haplotypes $(2,34,37,41-$ $43,47,49,51,56,58,62-70)$. However, T1D susceptibility haplotypes exist in a limited number. For instance, in Finland which has the highest incidence of T1D globally, only 37 different HLA haplotypes have been identified among diabetic children who had either a parent or sibling with T1D and another 18 haplotypes in children with first-degree relative who did not have T1D when potentially different HLA haplotypes in the global populations can be billions(71). 


\section{Table 1: Classification of HLA-DR alleles and their risk level}

\begin{tabular}{|c|c|c|c|c|c|}
\hline$H L A-$ & $D Q: A I$ & $D Q B 1$ & $D R B 1$ & Susceptibility & Populations \\
\hline$D R 2$ & 01:02 & 06:02 & $15: 01$ & Low risk & Almost all \\
\hline$D R 2$ & 01:02 & 05:02 & 16:01 & Moderate Risk & Caucasians \\
\hline$D R 2$ & 01:03 & 06:01 & $15: 02$ & Neutral & $\begin{array}{l}\text { Caucasians, Saudi } \\
\text { Arabia, Algeria }\end{array}$ \\
\hline DR3 & 05:01 & 02:01 & 03:01 & High Risk & $\begin{array}{l}\text { Caucasians, Koreans, } \\
\text { Bahraini } \\
\text { Kuwaiti } \\
\text { Egyptians } \\
\end{array}$ \\
\hline DR4 & 03:01 & 03:02 & 04:01 & High Risk & Caucasians \\
\hline$D R 4$ & 03:01 & 03:02 & 04:02 & Moderate Risk & Caucasians \\
\hline$D R 4$ & 03:01 & 03:02 & 04:03 & Neutral & Caucasians \\
\hline DR4 & 03:01 & 03:02 & 04:04 & Moderate Risk & Caucasians \\
\hline DR4 & 03:01 & 03:02 & 04:05 & High Risk & $\begin{array}{l}\text { Caucasians } \\
\text { Saudi Arabia } \\
\text { Algerian }\end{array}$ \\
\hline$D R 4$ & 03:01 & 03:01 & 04:01 & Neutral & Caucasians \\
\hline$D R 4$ & 03:01 & 03:03 & 04:01 & Neutral & Caucasians \\
\hline$D R 4^{*}$ & 04:05 & 03:03 & 04:01 & High Risk & Japanese, Koreans \\
\hline$D R 7$ & 02:01 & 03:03 & 07:01 & Low Risk & Caucasians \\
\hline DR6 & 01:01 & 05:03 & 04:01 & Low Risk & Caucasians \\
\hline$D R 8^{*}$ & 08:02 & 03:01 & 03:02 & High Risk & Japanese \\
\hline$D R 9^{*}$ & 09:01 & 03:00 & 03:03 & High Risk & Japanese, Koreans \\
\hline$D R 13^{*}$ & 13:02 & 01:02 & $06 ; 04$ & High Risk & Japanese \\
\hline
\end{tabular}

*They are only found on Asians and not in Caucasians

There are only a few HLA studies conducted in Arab countries that compare their contribution to the rise of T1D globally, Table 2 [3,48-54]. Most available studies have not used systematic HLA research standards. They have discussed HLA association randomly on either allele based or haplotypes $(38,46-54)$. Nevertheless, these studies have taken the first steps to elucidate genetic risk factors in the Arab population $(49,58,64,67,72)$. The hallmark of HLA susceptibility is, however, to be considered from the haplotype point of view $(4,25,34,35,37,39,41-43,47-49,51-56,58,60,64,66-79)$. Hamza et al 2015 have analyzed 23,333 articles, of which, only 30 were based on an Arab population (34). These studies mainly discussed genetic susceptibility of T1D related to HLA-DR or DQ alleles but not haplotype configurations. Hamzeh et al reported that Arab HLA indicate that 80 $\%$ of patients with T1D are carriers of DR3 or DR4, typical in Caucasoid populations (34). In addition, HLA-DR3/DR4 which present the highest diabetes risk is reported in 13\% $75 \%$ patients with $\mathrm{T} 1 \mathrm{D}(80)$.

Furthermore, it has been suggested that the presence of DR9 and low proportion of DR4 haplotype is an important factor in the low rate T1D within the Japanese population $(2,63,78)$. In fact, the variation in HLA-DR locus in HLA haplotypes in heterogenetic populations may in part explain the differences in T1D worldwide. However, this variation is not fully understood, since only limited comparisons of HLA haplotypes between populations are available $(56,81,82)$.

In fact, the same is true in some neighboring countries in the Middle East and North African (MENA) region . For example, in Iran it was reported that HLA DRB1* 03:01/ DQA1 $^{*}$ 05:01/DQB1* $02: 01$ are conferring a high risk of T1D(83). Sayad et al. reported that 
HLA DRB1*04:01, DQB1*03:02 alleles and DRB1*04:01- DQB1*03:02 haplotypes were significantly more common in male than female T1D patients and that DRB1*03:01, DRB1*15:01, DQB1*06:01 alleles, DQB1*03:01/05:01 genotype, DRB1*03:01-DQB1*02:01 and DRB1*15:01-DQB1*06:01 haplotypes were significantly greater in the female than male T1D patients. They also reported that the latter HLA haplotypes were significantly associated with age at onset of T1D. A strong association in age of onset between members of sib pairs was observed when the analysis was performed using contingency tables that split sibs into three age-at-onset ranges(84). Data from Turkey confirms similar trends in the distribution of T1D HLA susceptibility alleles as seen in other Caucasoid populations(79).

The ICA is considered a composite of specific anti $\beta$-cell antibodies, several of which have now been characterized at the molecular level (85) i.e. IAA, GAD, IA-2, and ZnT-8.

$\beta$-cell antibodies can be used to study the natural history of the preclinical phase of T1D, identify individuals at high risk, and thus for instance select such individuals for trials on immune intervention. The risk of developing clinical disease increases dramatically with an increase in the number of antibodies; it increased to 70-90\% in the presence of two and three antibodies(85). There is a dearth amount of information about autoantibodies in T1D in Arab populations (Table 2). Majority of the published studies have used the case-control design and investigated the presence of only GADA and IA2 $(58,86-89)$. There are interesting studies correlating gene polymorphisms and the ICA, IA2 and GADA to the susceptibility to T1D in the Kuwaiti population $(58,89)$. These findings may be confounded by variation in assay standardization and the number of auto antibodies measured. The current incomplete understanding of autoantibody profiles of T1D in Arab populations stresses the urgent need to study the nature of the massive increase of T1D in Arabs. Characterization of Arab T1D according to autoantibody profiles and HLA typing will be the base for all our future studies. No doubt any future intervention or cure will be based on the latter characterization.

Table 2: Number of anti-islet autoantibody measured in different Arab population studies

\begin{tabular}{|c|c|c|c||}
\hline \hline IAA & IA2 & GAD & ZnT8 \\
\hline \hline$\checkmark$ & $\checkmark$ & $\checkmark$ & $\checkmark$ \\
\hline \hline$\checkmark$ & $\checkmark$ & $\checkmark$ & - \\
\hline \hline- & $\checkmark$ & $\checkmark$ & - \\
\hline
\end{tabular}

Although IAA is more frequent in children, it has not been included in majority of studies.

Many studies have been performed identifying associations not only in genes constituting the HLA region but also outside this region (58). In order to study genetic risk in T1D genome-wide association studies (GWAS) have revolutionized genetic studies in search of genetic risk factors. GWAS enables to investigate a genome in search of causal variants, variants that contribute to an onset of a disease but may not carry enough susceptibility for this disease in isolation(64). Analyses performed by The Welcome Trust Case Control Consortium (WTCCC)(90) and Type 1 Diabetes Genetics Consortium have revealed more than 50 genetic variants associated with T1D (91). However, none of the non-HLA susceptibility loci were not even close to the risk associated with HLA. 


\section{Other Candidate genes for T1D}

Prior to GWAS only five loci were determined to be associated with T1D, HLA region, insulin (INS), cytotoxic T-lymphocyte-associated protein 4 (CTLA4), protein tyrosine phosphatases (PTPN22) and Interleukin 2 receptor alpha (IL2RA). Majority of the genes identified by GWAS meta-analyses can act as modulators of an immune-related process through both antigen presentation and other modifications of the immune function(91). A number of candidate genes involved in T1D are known as modulators of $\beta$-cell apoptosis, viral infection or islet inflammation. Some of the SNPs associated with a variation in susceptibility to T1D affect changes in gene expression (genetic variants in regulatory elements of genes can result in alteration of transcription and then gene expression). In addition, most of the identified susceptibility genes are expressed both in immunecompetent cells and in pancreatic $\beta$-cells, which suggests a genetically modulated dialogue between these two components of $\mathrm{T} 1 \mathrm{D}(91)$.

\section{T1D Natural History}

The natural history of T1D was hypothesized by late George Eisenbarth in 1986 (92); as typical for complex diseases both environmental and genetic factors are contributing for the disease development(93). The disease onset is triggered in genetically susceptible individuals to factors that are thus far not known(94). Tremendous biochemical reactions are encountered during initiation of $\beta$-cell mass autoimmunity in the preclinical phase until the actual onset of the disease manifestations (Figure 1). Different anti-islet autoantibodies, IA2, IAA, GADA and ZnT8, glucose intolerance arising from further damage and loss of $\beta$-cell function, and ultimately culminates with clinical signs and symptoms of diabetes $(61,62,93-95)$. Identification of the latter stage(s) in early phase of preclinical of disease prior to destruction of substantial amounts of $\beta$-cells by the immune system is critically important $(2,13,30,96,97)$. Any manipulation at the preclinical stages of the disease a delay of clinical phase and eventually cure might be possible. Insel and colleagues have recognized three different phases of human T1D, which allows for interventions designed to delay and, ultimately, prevent the onset of clinical symptoms. However, genetic susceptibility and triggering factors that were accounted for in these current phases were based almost solely on American and European studies(96) .

Recently, Pozzili et al. have suggested "re-structuring the natural history of T1D"(98) based on recent two findings using a novel technique to analyze pancreatic tissue obtained from 12 organ donors with T1D of varying disease durations, including some at onset, and some tissue obtained from previously healthy organ donors. Imaging mass cytometry was used, a recently developed technique that combines laser ablation with mass cytometry, permitting simultaneous investigation of up to 50 biomarkers from tissue sections stained with metal-labelled antibodies $(99,100)$. With this technique it might be possible to dissect islet cell morphology simultaneously with the characterization of infiltrating immune cells as well as different $\beta$-cell proteins. The authors reported that in some pancreatic tissue at the time of T1D onset, the proportion of $\beta$-cells approaches that observed in healthy pancreas, therefore indicating that at diagnosis there are still plenty of $\beta$-cells to be potentially protected from destruction before disease progression. This finding was already suggested long ago but now finds a definitive confirmation. By contrast, in pancreas from T1D donors with a longer disease duration ( $>8$ years), no $\beta$-cells were detected.

Taken together, these observations reinforce the concept of the large heterogeneity of T1D that manifests not only clinically but also at the histopathological level. Another important finding in these studies is the temporal correlation between $\beta$-cell destruction and immune cell infiltration in the islets (known as insulitis). In both papers, cytotoxic $\mathrm{T}$ cells, helper T cells, B cells, monocytes and macrophages were all detected in pancreatic islets and they appeared almost simultaneously with a change in $\beta$-cell phenotype. This finding might indicate that the initial event in T1D consists of insulitis to which $\beta$-cells react by losing surface expression of specific markers and losing the capacity for insulin production. It can be hypothesized that $\beta$-cells do change their phenotype (via loss of 
specific markers) as an attempt to escape from immune attack in a sort of 'immunological camouflage. As the disease progresses and $\beta$-cells remain alive, the immune-mediated attack persists. The modified $\beta$-cell phenotype might allow escape from detection by immune cells, which offers new possibilities for therapeutic intervention.

\section{Discussion}

The registration of T1D cases is inadequate in the GCC, although it could be easily set up since all countries have national free health care systems and many healthcare practitioners and researchers argue that incidence of the disease is rising sharply. The rising incidence of T1D in the GCC is mainly due to improved childhood mortality, rapid lifestyle changes, as well as "change in nutrition", especially in the early years, changes in breastfeeding practices, environmental pollutants and toxins, immune deficiency caused by greater hygienic standards and low vitamin D levels, which are highly prevalent in the region in spite of the sunshine." In people with T1D in the GCC countries, the characterization and identification of the HLA haplotypes associated with the disease and the determination of pancreatic islet autoantibodies as the present biomarkers for $\beta$-cell destruction will make it possible to develop a scientifically sound prediction algorithm. Screening among first-degree (and second-degree) relatives of people with T1D can help to identify family members who are at risk of acquiring the disease and to improve early management of the disease. On the other hand, we must recognize that the penetrance of genetic predisposition is incomplete as shown by twin studies revealing that less than $50 \%$ of cotwins of a twin with T1D will develop the disease (101). Thus, many people in the population transmit the genetic predisposition without having T1D themselves; thus, there are many "sporadic" cases of T1D. In addition to the lower risk of ketoacidosis at the preclinical phase, there might be a value from earlier therapeutic interventions, when some amount of functioning $\beta$-cells still exist and to preserve them, while at the clinical phase after diagnosis many $\beta$-cells will be destroyed or damaged. The careful registration of T1D cases, prediction and intervention are required in order to stop it from spiraling out of control.

In the three GCC countries were published data are available, i.e. Kuwait, Saudi Arabia, and Qatar T1D has shown an exponentially increasing incidence rates. There is paucity of reports on HLA as the major genetic susceptibility predictor and on anti-islet autoantibodies in the GCC countries. Novel concepts on the rate and degree of $\beta$-cell loss throughout the natural history of T1D have been put forward to aid in understanding the natural history of the disease. Descriptive research is needed to improve T1D management and adjustment in young children and their caregivers, behavioral or educational programs to the patients and their families. Targeted interventions for young children with T1D and their caregivers are needed to reduce the risks for long-term T1D-related complications and improve more immediate T1D management and quality of life. A systematic study among GCC Arab populations on HLA and anti-islet autoantibody profiling is essential to add the currently missing piece in the jigsaw part of global T1D etiology.

Concluding Remarks

- T1D is growing in an alarming manner in the GCC,

- T1D genotypes and phenotypes are not well characterized in the GCC,

- $\quad$ Guidelines and procedures for diagnosis of T1D (islet Autoantibody profiling and HLA typing) are needed,

- Misdiagnosis of LADA and T2D can happen,

- $\quad$ Suitable infrastructure for scientifically sound systematic scientific research on T1D is urgently needed,

- Healthcare policy makers are invited to laydown proper policies and procedures to encourage commissioning of GCC T1D consortium to utilize the above facilities and generous available funds to establish proper GCC-T1D registry and research should be established, 
- Any tiny alteration genetically, biochemically or environmentally will have significant impacts in the world understanding of T1D etiology.

\begin{abstract}
Author Contributions: Conceptualization, methodology, validation, formal analysis, investigation and writing-original draft preparation, M.J.; review and editing, M.J., M.SH., J.T.; visualization, M.J., M.SH., J.T.; supervision, M.J.; project administration, M.J. All authors have read and agreed to the published version of the manuscript.

Funding: "This research received no external funding."

Conflicts of Interest: “The authors declare no conflict of interest.
\end{abstract}

\title{
References
}

1. Mohamed M Jahromi, George S Eisenbarth. Genetic determinants of type 1 diabetes across populations. Annals of the New York Academy of Sciences. 2006;1079: 289-299. doi:10.1196/ANNALS.1375.044

2. Jahromi MM, Eisenbarth GS. Genetic determinants of type 1 diabetes across populations. Annals of the New York Academy of Sciences. 2006. doi:10.1196/annals.1375.044

3. Knip M, Siljander H. Autoimmune mechanisms in type 1 diabetes. Autoimmunity Reviews. 2008;7: 550-557. doi:10.1016/J.AUTREV.2008.04.008

4. Nejentsev S, Howson JMM, Walker NM, Szeszko J, Field SF, Stevens HE, et al. Localization of type 1 diabetes susceptibility to the MHC class I genes HLA-B and HLA-A. Nature. 2007;450: 887. doi:10.1038/NATURE06406

5. Theresa A Aly, Erin E Baschal, Mohamed M Jahromi, Maria S Fernando, Sunanda R Babu, et al. Analysis of single nucleotide polymorphisms identifies major type $1 \mathrm{~A}$ diabetes locus telomeric of the major histocompatibility complex. Diabetes. 2008;57: 770-776. doi:10.2337/DB07-0900

6. Bergholdt R, Brorsson C, Palleja A, Berchtold LA, Fløyel T, Bang-Berthelsen CH, et al. Identification of Novel Type 1 Diabetes Candidate Genes by Integrating Genome-Wide Association Data, Protein-Protein Interactions, and Human Pancreatic Islet Gene Expression. Diabetes. 2012;61: 954. doi:10.2337/DB11-1263

7. Jill M Norris, Randi K Johnson, Lars C Stene. Type 1 diabetes-early life origins and changing epidemiology. The lancet Diabetes \& endocrinology. 2020;8: 226-238. doi:10.1016/S2213-8587(19)30412-7

8. Saraswathi S, Al-Khawaga S, Elkum N, Hussain K. A Systematic Review of Childhood Diabetes Research in the Middle East Region. Frontiers in Endocrinology. 2019;10. doi:10.3389/FENDO.2019.00805

9. David M Nathan, Patricia A Cleary, Jye-Yu C Backlund, Saul M Genuth, John M Lachin, et al. Intensive diabetes treatment and cardiovascular disease in patients with type 1 diabetes. The New England journal of medicine. 2005;353: 2643-2653. doi:10.1056/NEJMOA052187

10. Abdulaziz Al Dawish M, Alwin Robert A, Braham R, Abdallah Al Hayek A, al Saeed A, Ahmed Ahmed R, et al. Diabetes Mellitus in Saudi Arabia: A Review of the Recent Literature. Current Diabetes Reviews. 2016;12: 359-368.

doi:10.2174/1573399811666150724095130

11. IDF Diabetes Atlas 9th edition 2019. [cited 1 Aug 2021]. Available: https://www.diabetesatlas.org/en/ 
12. Azza A Shaltout, Deborah Wake, Thangavel A Thanaraj, Dina M Omar, Dalia Al-AbdulRazzaq, et al. Incidence of type 1 diabetes has doubled in Kuwaiti children 0-14 years over the last 20 years. Pediatric diabetes. 2017;18: 761-766. doi:10.1111/PEDI.12480

13. Jahromi M, Al-Mulla F, Al-Ozairi E. Autoimmune signatures for prediction and diagnosis of autoimmune diabetes in Kuwait. Autoimmunity Reviews. 2019;18: 642-644. doi:10.1016/J.AUTREV.2019.02.009

14. G.D. OGLE, J. LESLEY, P. SINE AND P. MCMASTER. Type 1 diabetes mellitus in children in Papua New Guinea. Papua and New Guinea Medical Journal. 2001;44: 96-100. Available: http://europepmc.org/article/MED/12422979

15. Robert AA, Al-Dawish A, Mujammami M, Dawish MA. Type 1 Diabetes Mellitus in Saudi Arabia: A Soaring Epidemic. International Journal of Pediatrics. 2018;2018: 1-9. doi:10.1155/2018/9408370

16. $\mathrm{A} \mathrm{H}, \mathrm{MK}$ B, TW J, EA D. Incidence of childhood onset type 1 diabetes in Western Australia from 1985 to 2016: Evidence for a plateau. Pediatric diabetes. 2018;19: 690-692. doi:10.1111/PEDI.12636

17. Roche E, McKenna A, Ryder K, ... AB-E journal of, 2016 undefined. Is the incidence of type 1 diabetes in children and adolescents stabilising? The first 6 years of a National Register. Springer. [cited 30 Jul 2021]. Available: https://link.springer.com/article/10.1007/s00431-016-2787-6

18. Incidence and trends of childhood Type 1 diabetes worldwide 1990-1999. Diabetic Medicine: a journal of the British Diabetic Association. 2006;23: 857-866. doi:10.1111/J.1464-5491.2006.01925.X

19. Sex difference in the incidence of insulin-dependent diabetes mellitus: an analysis of the recent epidemiological data. World Health Organization DIAMOND Project Group - PubMed. [cited 1 Aug 2021]. Available: https://pubmed.ncbi.nlm.nih.gov/9509279/

20. Patterson CC, Harjutsalo V, Rosenbauer J, Neu A, Cinek O, Skrivarhaug T, et al. Trends and cyclical variation in the incidence of childhood type 1 diabetes in 26 European centres in the 25 year period 1989-2013: a multicentre prospective registration study. [cited $30 \mathrm{Jul} 2021$ ]. doi:10.1007/s00125-018-4763-3

21. Incidence of childhood-onset insulin-dependent diabetes mellitus: the EURODIAB ACE Study - Green A. [cited 2 Aug 2021]. Available: https://orbilu.uni.lu/handle/10993/27233

22. Satti SA, Saadeldin IY, Dammas AS. Diabetic Ketoacidosis in children admitted to Pediatric Intensive Care Unit of King Fahad Hospital, Al-Baha, Saudi Arabia: Precipitating factors, epidemiological parameters and clinical presentation. Sudanese journal of paediatrics. 2013;13: 24-30. Available: http://www.ncbi.nlm.nih.gov/pubmed/27493370

23. Al Rashed AM. Pattern of presentation in type 1 diabetic patients at the diabetes center of a university hospital. Annals of Saudi Medicine. 2011;31: 243-249.

24. Al-Dabbagh TQ. Epidemiology of type 1 diabetes mellitus among Arab children [2]. Saudi Medical Journal. 2006;27: 916.

25. Jahromi M, Rahma Z, Taha N, Lin P-Y, Yang K-L. Detection of the HLA-DRB4*01:44 allele in a Kuwaiti individual. HLA. 2020;96: 537-539. doi:10.1111/TAN.13981 
26. Detection of the HLA-DRB4*01:44 allele in a Kuwaiti individual - Jahromi - 2020 - HLA - Wiley Online Library. [cited 2 Aug 2021]. Available: https://onlinelibrary.wiley.com/doi/10.1111/tan.13981

27. Jahromi M, Al-Ozairi E. Human Leukocyte Antigen (HLA) and Islet Autoantibodies Are Tools to Characterize Type 1 Diabetes in Arab Countries: Emphasis on Kuwait. Disease Markers. 2019;2019.

28. Asirvatham Alwin Robert, Abdulrahman Al-Dawish,Muhammad Mujammami, and Mohamed Abdulaziz Al Dawish. Type 1 Diabetes Mellitus in Saudi Arabia: A Soaring Epidemic. International journal of pediatrics. 2018;2018: 1-9. doi:10.1155/2018/9408370

29. Bener A, Mohammad RR. Global distribution of consanguinity and their impact on complex diseases: Genetic disorders from an endogamous population. Egyptian Journal of Medical Human Genetics. 2017;18: 315-320. doi:10.1016/J.EJMHG.2017.01.002

30. Jahromi MM, Eisenbarth GS. Cellular and molecular pathogenesis of type 1A diabetes. Cellular and Molecular Life Sciences. 2007;64. doi:10.1007/s00018-007-6469-4

31. TUOMILEHTO-WOLF E, TUOMILEHTO J. Is the high incidence of diabetes in young children diagnosed under the age of 4 years determined by genetic factors in Finland ? Diabète et métabolisme (Paris). 1993;19: 167-172.

32. Cudworth A, Lancet JW-T, 1974 undefined. HL-A antigens and diabetes mellitus. thelancet.com. [cited 30 Jul 2021 ]. Available: https://www.thelancet.com/journals/lancet/article/PIIS0140-6736(74)90930-1/fulltext

33. Nerup J, Platz P, Andersen OO, Christy M, Lyngsoe J, et al. HL-A antigens and diabetes mellitus. Lancet (London, England). 1974;2: 864-866. doi:10.1016/S0140-6736(74)91201-X

34. A. R. Hamzeh, P. Nair, N. Al-Khaja, M. T. Al Ali. Association of HLA-DQA1 and -DQB1 alleles with type I diabetes in Arabs: a meta-analyses. Tissue antigens. 2015;86: 21-27. doi:10.1111/TAN.12598

35. Carreno BM, Anderson RW, Coligan JE, Biddison WE. HLA-B37 and HLA-A2.1 molecules bind largely nonoverlapping sets of peptides. Proceedings of the National Academy of Sciences of the United States of America. 1990;87: 3420. doi:10.1073/PNAS.87.9.3420

36. MJ R, AK S, A P. Genetics of type 1 diabetes. Pediatric diabetes. 2018;19: 346-353. doi:10.1111/PEDI.12597

37. Tuomilehto-Wolf E, Tuomilehto J. HLA antigens in insulin-dependent diabetes mellitus. Annals of Medicine. 1991;23: 481-488. doi:10.3109/07853899109150507

38. Valdes AM, Erlich HA, Noble JA. Human Leukocyte Antigen Class I B and C Loci Contribute to Type 1 Diabetes (T1D) Susceptibility and Age at T1D Onset. Human immunology. 2005;66: 301. doi:10.1016/J.HUMIMM.2004.12.001

39. Clara Gorodezkya, Carmen Alaeza, Andrea Murguíaa, Araceli Rodrígueza, Sandra Balladaresa, et al. HLA and autoimmune diseases: Type 1 diabetes (T1D) as an example. Autoimmunity reviews. 2006;5: 187-194. doi:10.1016/J.AUTREV.2005.06.002

40. Jahromi M, Al-Ozairi E. Human Leukocyte Antigen (HLA) and Islet Autoantibodies Are Tools to Characterize Type 1 Diabetes in Arab Countries: Emphasis on Kuwait. Disease Markers. 2019;2019. doi:10.1155/2019/9786078 
41. Al-Khuwari M, Al-Khuwari M, Al-Khuwari M. HLA-DQB1*04:02:13, an HLA-DQB1*04:02 variant, identified in a Kuwaiti individual. HLA. 2019;95: 80-81. doi:10.1111/tan.13714

42. Jahromi M, Jacob SP, Taha N, Lin PY, Yang KL. Identification of HLA-C*16:46 allele in a Kuwaiti individual. HLA. 2020;95: 71-72. doi:10.1111/TAN.13705

43. Jahromi M, Rahma Z, Nazim R, Lin P-Y, Yang K-L. Recognition of an HLA-C*04 variant, HLA-C*04:30, in two Kuwaiti families. HLA. 2019;94: 452-453. doi:10.1111/TAN.13669

44. Aly TA, Baschal EE, Jahromi MM, Kretowski A, Babu SR, Rewers MJ, et al. High Density SNP Analysis of the MHC Region Reveals Multiple Loci for Type 1A Diabetes. Clinical Immunology. 2007;123. doi:10.1016/j.clim.2007.03.018

45. Barrett J, Clayton D, Concannon P, ... BA-N, 2009 undefined. Genome-wide association study and meta-analysis find that over 40 loci affect risk of type 1 diabetes. nature.com. [cited 30 Jul 2021]. Available: https://www.nature.com/articles/ng.381

46. Noble JA, Erlich HA. Genetics of Type 1 Diabetes. [cited 30 Jul 2021]. doi:10.1101/cshperspect.a007732

47. Noble JA, Johnson J, Lane JA, Valdes AM. Race-specific type 1 diabetes risk of HLA-DR7 haplotypes. Tissue Antigens. 2011;78: 348-351. doi:10.1111/J.1399-0039.2011.01772.X

48. Kawabata Y, Ikegami H, Kawaguchi Y, Diabetes TF-, 2002 undefined. Asian-specific HLA haplotypes reveal heterogeneity of the contribution of HLA-DR and-DQ haplotypes to susceptibility to type 1 diabetes.. Am Diabetes Assoc. [cited 30 Jul 2021]. Available: https://diabetes.diabetesjournals.org/content/51/2/545.short

49. Mouna Stayoussef, Jihen Benmansour, Fayza A. Al-Jenaidi, Rita Nemr, Muhallab E. Ali, et al. Influence of common and specific HLA-DRB1/DQB1 haplotypes on genetic susceptibilities of three distinct Arab populations to type 1 diabetes. Clinical and vaccine immunology : CVI. 2009;16: 136-138. doi:10.1128/CVI.00215-08

50. Ken B Hanscombe, David L Morris, Janelle A Noble, Alexander T Dilthey, Philip Tombleson, et al. Genetic fine mapping of systemic lupus erythematosus MHC associations in Europeans and African Americans. Human molecular genetics. 2018;27: 3813-3824. doi:10.1093/HMG/DDY280

51. Baschal EE, Aly TA, Babu SR, Fernando MS, Yu L, Miao D, et al. HLA-DPB1*0402 Protects Against Type 1A Diabetes Autoimmunity in the Highest Risk DR3-DQB1*0201/DR4-DQB1*0302 DAISY Population. Diabetes. 2007;56: 24052409. doi:10.2337/DB07-0029

52. Noble JA, Valdes AM. Genetics of the HLA Region in the Prediction of Type 1 Diabetes. Current diabetes reports. 2011;11: 533. doi:10.1007/S11892-011-0223-X

53. Janelle A Noble, Jameel Johnson, Julie A Lane, Ana M Valdes. HLA class II genotyping of African American type 1 diabetic patients reveals associations unique to African haplotypes. Diabetes. 2013;62: 3292-3299. doi:10.2337/DB130094

54. Noble JA, Johnson J, Lane JA, Valdes AM. Race-specific type 1 diabetes risk of HLA-DR7 haplotypes. Tissue Antigens. 2011;78: 348-351. doi:10.1111/J.1399-0039.2011.01772.X 
55. Lipner EM, Tomer Y, Noble JA, Monti MC, Lonsdale JT, Corso B, et al. Linkage Analysis of Genomic Regions Contributing to the Expression of Type 1 Diabetes Microvascular Complications and Interaction with HLA. Journal of Diabetes Research. 2015;2015. doi:10.1155/2015/694107

56. Henry Erlich, Ana Maria Valdes, Janelle Noble, Joyce A Carlson, Mike Varney, et al. HLA DR-DQ haplotypes and genotypes and type 1 diabetes risk: analysis of the type 1 diabetes genetics consortium families. Diabetes. 2008;57: 10841092. doi:10.2337/DB07-1331

57. Onengut-Gumuscu S, Chen W-M, Robertson CC, Bonnie JK, Farber E, Zhu Z, et al. Type 1 Diabetes Risk in African-Ancestry Participants and Utility of an Ancestry-Specific Genetic Risk Score. Diabetes Care. 2019;42: 406-415. doi:10.2337/DC18-1727

58. Haider MZ, Rasoul MA, Al-Mahdi M, Al-Kandari H, Dhaunsi GS. Association of protein tyrosine phosphatase non-receptor type 22 gene functional variant C1858T, HLA-DQ/DR genotypes and autoantibodies with susceptibility to type-1 diabetes mellitus in Kuwaiti Arabs. PLOS ONE. 2018;13: e0198652. doi:10.1371/JOURNAL.PONE.0198652

59. N Jassam, N Amin, P Holland, R K Semple, D J Halsall, G Wark, and J H Barth. Analytical and clinical challenges in a patient with concurrent type 1 diabetes, subcutaneous insulin resistance and insulin autoimmune syndrome. Endocrinology, diabetes \& metabolism case reports. 2014;2014. doi:10.1530/EDM-13-0086

60. J M M Howson, N M Walker, D Clayton, J A Todd, Type 1 Diabetes Genetics Consortium. Confirmation of HLA class II independent type 1 diabetes associations in the major histocompatibility complex including HLA-B and HLA-A. Diabetes, obesity \& metabolism. 2009;11 Suppl 1: 31-45. doi:10.1111/J.1463-1326.2008.01001.X

61. Jennifer M Barker, Taylor M Triolo, Theresa A Aly, Erin E Baschal, Sunanda R Babu, Adam Kretowski, et al. Two single nucleotide polymorphisms identify the highest-risk diabetes HLA genotype: potential for rapid screening. Diabetes. 2008;57: 3152-3155. doi:10.2337/DB08-0605

62. Aly TA, Ide A, Jahromi MM, Barker JM, Fernando MS, Babu SR, et al. Extreme genetic risk for type 1A diabetes. Proceedings of the National Academy of Sciences of the United States of America. 2006;103. doi:10.1073/pnas.0606349103

63. Zhang L, Gianani R, Nakayama M, Liu E, Kobayashi M, Baschal E, et al. Type 1 diabetes: Chronic progressive autoimmune disease. Novartis Foundation Symposium. 2008.

64. Mourad Aribi, Soraya Moulessehoul, Ahmed-Bakir Benabadji, Mohammed Kendoucitani. HLA DR phenotypic frequencies and genetic risk of Type 1 diabetes in west region of Algeria, Tlemcen. BMC genetics. 2004;5.

doi:10.1186/1471-2156-5-24

65. M Z Haider, A Shaltout, K Alsaeid, M Qabazard, J Dorman. Prevalence of human leukocyte antigen DQA1 and DQB1 alleles in Kuwaiti Arab children with type 1 diabetes mellitus. Clinical genetics. 1999;56: 450-456.

doi:10.1034/J.1399-0004.1999.560608.X

66. Jahromi M, Jacob SP, Taha N, Lin P-Y, Yang K-L. Identification of HLA-C*16:46 allele in a Kuwaiti individual. HLA. 2020;95: 71-72. doi:10.1111/TAN.13705 
67. Fekih Mrissa N, Mrad M, Ouertani H, Baatour M, Sayeh A, Nsiri B, et al. Association of HLA-DR-DQ polymorphisms with diabetes in Tunisian patients. Transfusion and Apheresis Science. 2013;49: 200-204.

68. Mosaad YM, Auf FA, Metwally SS, Elsharkawy AA, El-Hawary AK, Hassan RH, et al. HLA-DQB1* alleles and genetic susceptibility to type 1 diabetes mellitus. World J Diabetes. 2012;3: 149-155. doi:10.4239/wjd.v3.i8.149

69. Jabbar AAR, Mezaal TJ, Dawood FH. Association of HLA antigens with diabetes mellitus in an Iraqi population. Disease Markers. 1989;7: 79-85.

70. HLA associations in an Arab type 1 diabetic population - PubMed. [cited 2 Aug 2021]. Available: https://pubmed.ncbi.nlm.nih.gov/3502987/

71. Tienari PJ, Tuomilehto-Wolf E, Tuomilehto J, Peltonen L. HLA haplotypes in Type 1 (insulin-dependent) diabetes mellitus: molecular analysis of the HLA-DQ locus. Diabetologia. 1992.

72. Al-Jenaidi FA, Wakim-Ghorayeb SF, Al-Abbasi A, Arekat MR, Irani-Hakime N, Najm P, et al. Contribution of Selective HLA-DRB1/DQB1 Alleles and Haplotypes to the Genetic Susceptibility of Type 1 Diabetes among Lebanese and Bahraini Arabs. The Journal of Clinical Endocrinology \& Metabolism. 2005;90: 5104-5109. doi:10.1210/JC.2005-1166

73. Farina, Picascia, Pisapia, Barba, Vitale, Franzese, et al. HLA-DQA1 and HLA-DQB1 Alleles, Conferring Susceptibility to Celiac Disease and Type 1 Diabetes, are More Expressed Than Non-Predisposing Alleles and are Coordinately Regulated. Cells. 2019;8: 751. doi:10.3390/CELLS8070751

74. Mosaad YM. HLA-DQB1* alleles and genetic susceptibility to type 1 diabetes mellitus. World Journal of Diabetes. 2012;3: 149. doi:10.4239/WJD.V3.18.149

75. Tienari PJ, Tuomilehto-Wolf E, Tuomilehto J, Peltonen L, >kerblom HK, Fagerlund A, et al. HLA haplotypes in Type 1 (insulin-dependent) diabetes mellitus: molecular analysis of the HLA-DQ locus. Diabetologia. 35: 254-260. Available: https://www.academia.edu/16063609/HLA_haplotypes_in_Type_1_insulin_dependent_diabetes_mellitus_molecular_analysis_of_the_HLA_DQ_locus

76. Mustonen N, Siljander H, Peet A, Tillmann V, Härkönen T, Ilonen J, et al. Early childhood infections precede development of beta-cell autoimmunity and type 1 diabetes in children with HLA-conferred disease risk. Pediatric Diabetes. 2018;19: 293-299. doi:10.1111/PEDI.12547

77. Dorman JS, Bunker CH. HLA-DQ Locus of the Human Leukocyte Antigen Complex and Type 1 Diabetes Mellitus: A HuGE Review. Epidemiologic Reviews. 2000;22: 218-227. doi:10.1093/OXFORDJOURNALS.EPIREV.A018034

78. Nakanishi K, Inoko H. Combination of HLA-A24, -DQA1*03, and -DR9 Contributes to Acute-Onset and Early Complete $\beta$-Cell Destruction in Type 1 Diabetes. Diabetes. 2006;55: 1862-1868. doi:10.2337/DB05-1049

79. Mehmet Keskin, Ayşe Aygün, Sacide Pehlivan, Özlem Keskin, Yılmaz Kor, Ayşe Balat, Yavuz Coşkun. Trends in the frequency of HLA DR-DQ haplotypes among children and adolescents with type 1 diabetes mellitus in the Southeast Region of Turkey. Journal of clinical research in pediatric endocrinology. 2012;4: 189-192. doi:10.4274/JCRPE.768

80. E Wolf, K M Spencer, A G Cudworth. The genetic susceptibility to type 1 (insulin-dependent) diabetes: analysis of the HLA-DR association. Diabetologia. 1983;24: 224-230. doi:10.1007/BF00282704 
81. JAHROMI MM, EISENBARTH GS. Genetic Determinants of Type 1 Diabetes Across Populations. Annals of the New York Academy of Sciences. 2006;1079: 289-299. doi:10.1196/ANNALS.1375.044

82. Varney MD, Valdes AM, Carlson JA, Noble JA, Tait BD, Bonella P, et al. HLA DPA1, DPB1 Alleles and Haplotypes Contribute to the Risk Associated With Type 1 Diabetes. Diabetes. 2010;59: 2055-2062. doi:10.2337/DB09-0680

83. Derakhshan SM, Sehrig FZ, Sohrabi N, Shiva S, Baradaran B, Khaniani MS. The association between human leukocyte antigen class II DR3-DQ2 haplotype and type 1 diabetes in children of the east azerbaijan state of Iran. Iranian Red Crescent Medical Journal. 2015;17. doi:10.5812/IRCMJ.28380

84. Sayad A, Akbari MT, Pajouhi M, Mostafavi F, Kazemnejad A, Zamani M. Investigation The Role of Gender on The HLADRB1 and -DQB1 Association with Type 1 Diabetes Mellitus in Iranian Patients. Cell journal. - Bing. [cited 2 Aug 2021]. Available: https://www.bing.com/search?q=Sayad+A\%2C+Akbari+MT\%2C+Pajouhi+M\%2C+Mostafavi+F\%2C+Kazemnejad+A\%2C+Zamani+M.+Investigation+The+Role+of+Gender+on+The+HLA-DRB1+and+DQB1+Association+with+Type+1+Diabetes+Mellitus+in+Iranian+Patients.+Cell+journal.\&cvid=a4146864ff5f48469550bb1f59c3697b\&aqs=edge.1.69i57j69i59.2905j0j9\&FORM=ANAB01\&PC=U531

85. Janet M. Wenzlau \& John C. Hutton. Novel diabetes autoantibodies and prediction of type 1 diabetes. Current diabetes reports. 2013;13: 608-615. doi:10.1007/S11892-013-0405-9

86. Abdulrahman Al-Hussaini, Nimer Sulaiman, Musa Al-Zahrani, Ahmed Alenizi, Imad El Haj. High prevalence of celiac disease among Saudi children with type 1 diabetes: a prospective cross-sectional study. BMC gastroenterology. 2012;12. doi:10.1186/1471-230X-12-180

87. Noura Al-Hassani, Fares Chedid, Suha Hadi and Walid Kaplan. Prevalence of autoantibodies in type 1 diabetes patients and its association with the clinical presentation - UAE Eastern Region experience. Journal of pediatric endocrinology \& metabolism : JPEM. 2014;27: 1157-1159. doi:10.1515/JPEM-2013-0430

88. Al-Hakami AM. Pattern of thyroid, celiac, and anti-cyclic citrullinated peptide autoantibodies coexistence with type 1 diabetes mellitus in patients from Southwestern Saudi Arabia. Saudi Medical Journal. 2016;37: 386-391. doi:10.15537/SMJ.2016.4.13571

89. Rasoul MA, Haider MZ, Al-Mahdi M, Al-Kandari H, Dhaunsi GS. Relationship of four vitamin D receptor gene polymorphisms with type 1 diabetes mellitus susceptibility in Kuwaiti children. BMC Pediatrics 2019 19:1. 2019;19: 1-13. doi:10.1186/S12887-019-1448-0

90. Burton PR, Clayton DG, Cardon LR, Craddock N, Deloukas P, Duncanson A, et al. Genome-wide association study of 14,000 cases of seven common diseases and 3,000 shared controls. Nature. 2007;447: 661-678.

91. Pociot F, Lernmark Å. Genetic risk factors for type 1 diabetes. The Lancet. 2016;387: 2331-2339. doi:10.1016/S0140$6736(16) 30582-7$

92. Eisenbarth GS. Type I Diabetes Mellitus. http://dx.doi.org/101056/NEJM198605223142106. 2009;314: 1360-8. doi:10.1056/NEJM198605223142106 
93. Mark A Atkinson, George S Eisenbarth. Type 1 diabetes: new perspectives on disease pathogenesis and treatment. Lancet (London, England). 2001;358: 221-229. doi:10.1016/S0140-6736(01)05415-0

94. Mark A Atkinson, George S Eisenbarth, Aaron W Michels. Type 1 diabetes. Lancet (London, England). 2014;383: 6982. doi:10.1016/S0140-6736(13)60591-7

95. Taplin C, Barker J. Autoantibodies in type 1 diabetes. Autoimmunity. 2008;41: 11-18. doi:10.1080/08916930701619169

96. Richard A Insel, Jessica L Dunne, Mark A Atkinson, Jane L Chiang, Dana Dabelea, et al. Staging presymptomatic type 1 diabetes: a scientific statement of JDRF, the Endocrine Society, and the American Diabetes Association. Diabetes care. 2015;38: 1964-1974. doi:10.2337/DC15-1419

97. Jahromi M, Al-Ozairi E. Human Leukocyte Antigen (HLA) and Islet Autoantibodies Are Tools to Characterize Type 1 Diabetes in Arab Countries: Emphasis on Kuwait. Disease Markers. 2019;2019. doi:10.1155/2019/9786078

98. Paolo Pozzilli \& Alberto Signore. The reconstructed natural history of type 1 diabetes mellitus. Nature reviews Endocrinology. 2019;15: 256-257. doi:10.1038/S41574-019-0192-8

99. Nicolas Damond, Stefanie Engler, Vito R.T. Zanotelli, Denis Schapiro, Clive H. Wasserfall, et. A Map of Human Type 1 Diabetes Progression by Imaging Mass Cytometry. Cell metabolism. 2019;29: 755-768.e5.

doi:10.1016/J.CMET.2018.11.014

100. Wang YJ, Traum D, Schug J, Gao L, Liu C, Atkinson MA, et al. Multiplexed In Situ Imaging Mass Cytometry Analysis of the Human Endocrine Pancreas and Immune System in Type 1 Diabetes. Cell Metabolism. 2019;29: 769-783.e4. doi:10.1016/J.CMET.2019.01.003

101. Valma Hyttinen, Jaakko Kaprio, Leena Kinnunen, Markku Koskenvuo, Jaakko Tuomilehto. Genetic liability of type 1 diabetes and the onset age among 22,650 young Finnish twin pairs: a nationwide follow-up study. Diabetes. 2003;52: 1052-1055. doi:10.2337/DIABETES.52.4.1052. 
Figure 1: Natural History of T1D

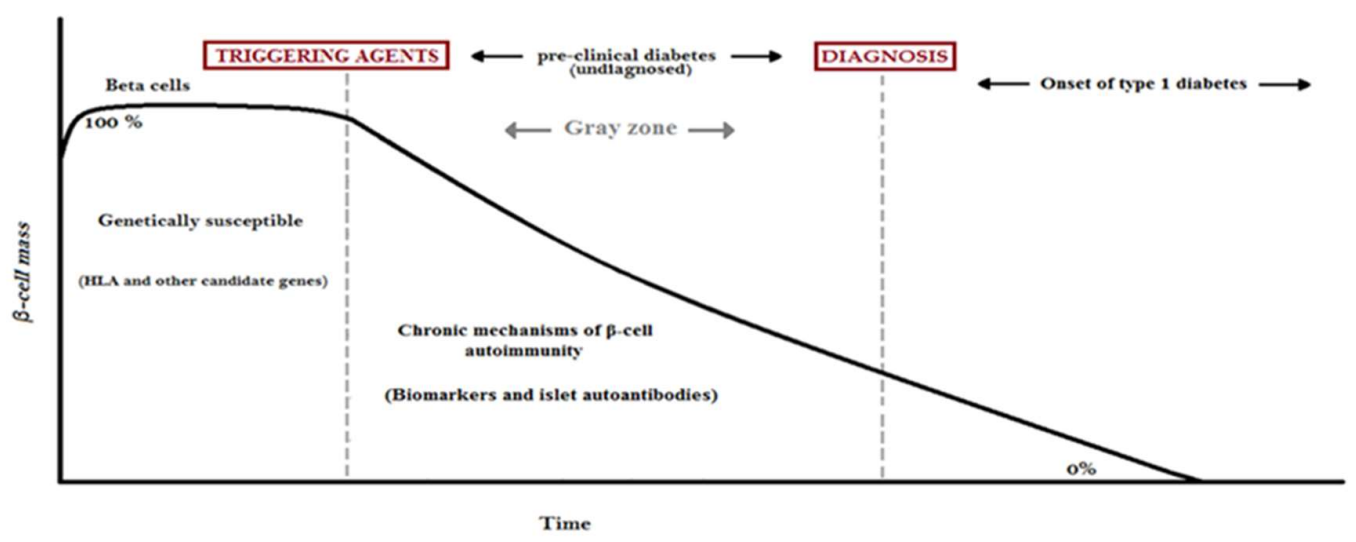

Illustration of natural history of T1D, reproduced by permission with slight modification for clarity in response to "Re-structuring Natural History of T1D" by Pozzili et al that after diagnosis still there is a small portion of $\beta$-cell mass is left, initial Natural History of T1D by Eisenbarth. Majority of biomarkers and islet autoantibodies develop after genetically susceptible individuals were exposed to certain triggering agents until the actual diagnosis of T1D, Gray Zone. 
Figure 2: Incidence Rate (IR) of T1D globally in countries where the data was available

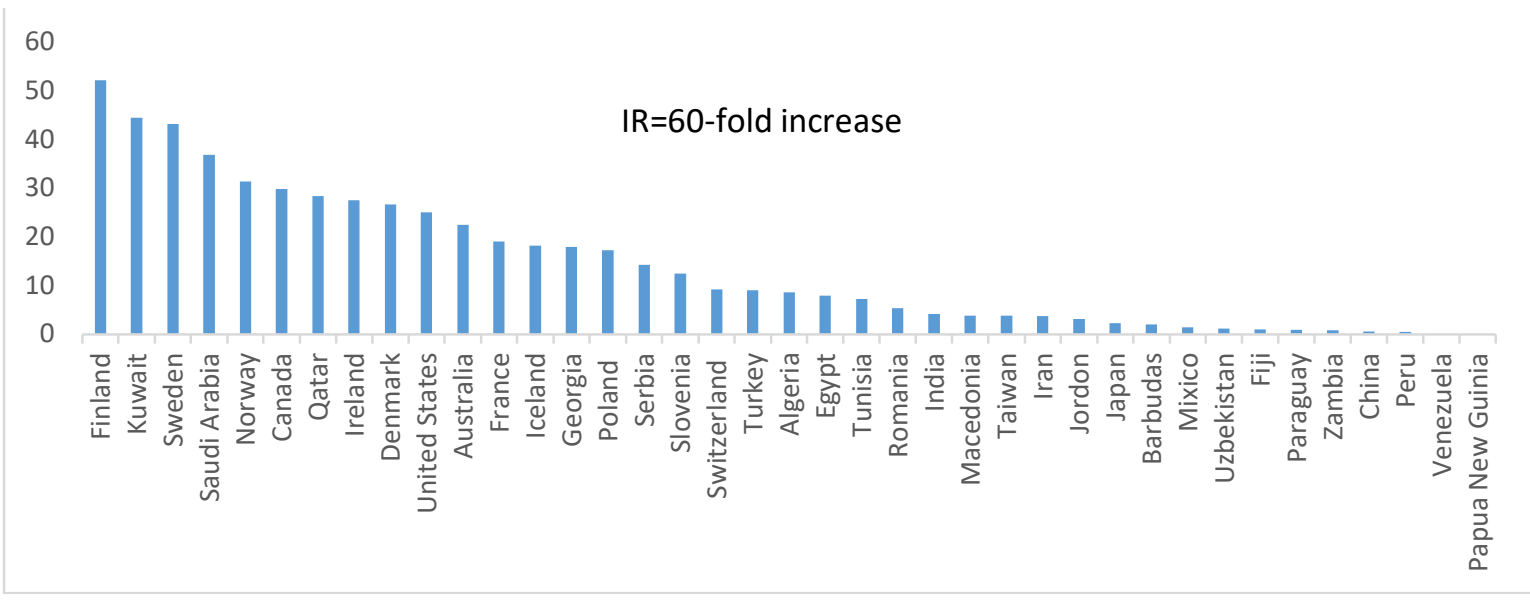

Illustration of IR of T1D in different countries globally. The IR varies from 52.7 in Finland down to 0.8 in Papua New Guinea with more than 60 folds increase. 
Figure 3: Illustration of IR of T1D in Arab Countries where data is available

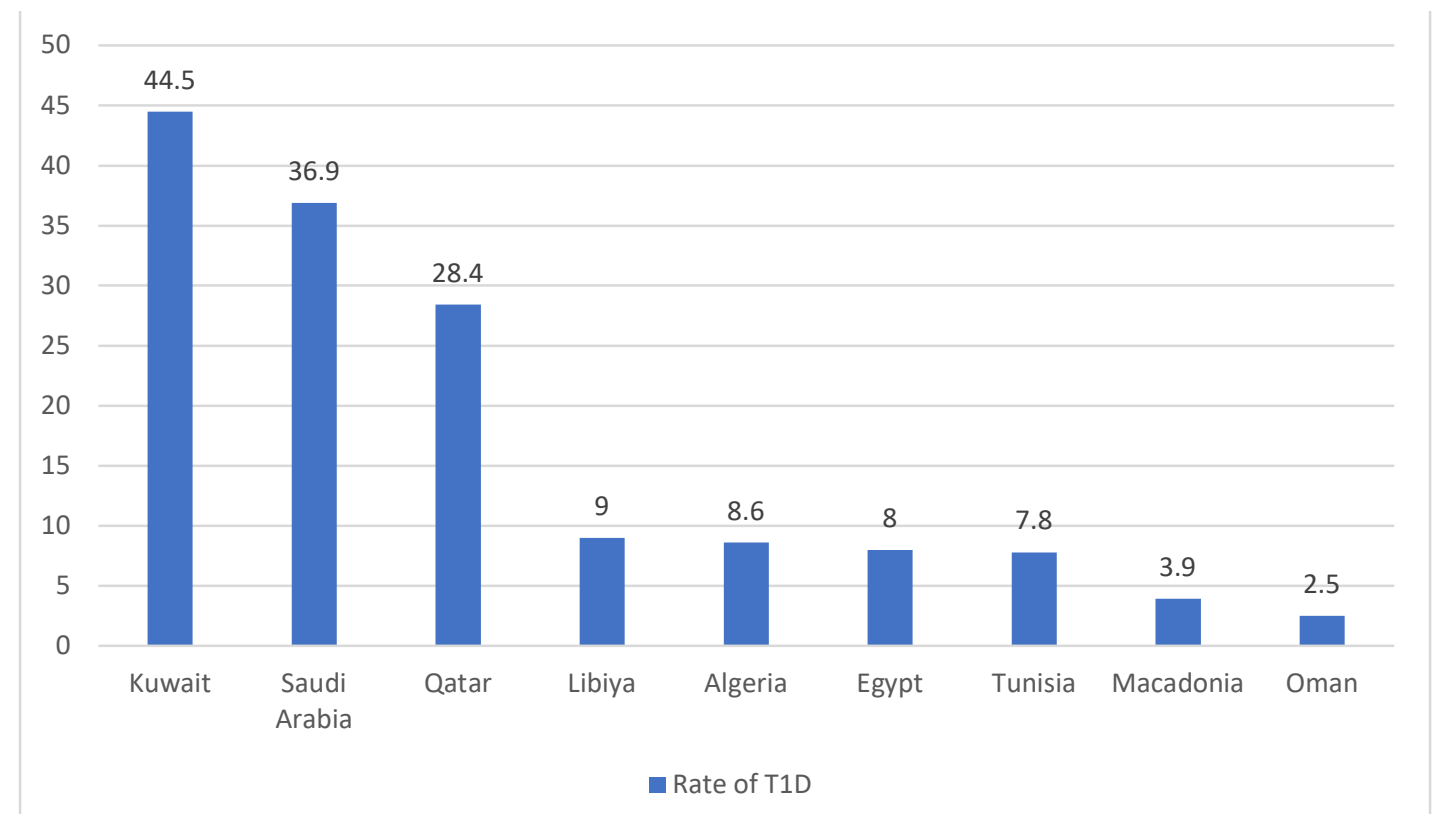

IR of T1D in Arab Countries varies significantly with 18-fold difference between Kuwait with 44.5 and Oman 2.5. The current situation, especially among the GCC counties with similar socioeconomical condition is alrming. 